\title{
Demographic, clinical, psychosocial, and environmental correlates of objectively assessed physical activity among breast cancer survivors
}

\author{
Caroline S Kampshoff ${ }^{1}$ • Fiona Stacey ${ }^{2}$ - Camille E Short ${ }^{3}$ - Willem van Mechelen ${ }^{1}$. \\ Mai JM Chinapaw ${ }^{1}$ • Johannes Brug ${ }^{4} \cdot$ Ronald Plotnikoff $^{5}$ • Erica L James ${ }^{2}$. \\ Laurien M Buffart ${ }^{4}$
}

Received: 13 October 2015 / Accepted: 22 February 2016/Published online: 12 March 2016

(C) The Author(s) 2016. This article is published with open access at Springerlink.com

\begin{abstract}
Purpose The aim of this study was to identify demographic, clinical, psychosocial, and environmental correlates of objectively assessed physical activity among breast cancer survivors.

Methods Baseline data were utilized from 574 female breast cancer survivors who participated in three different intervention studies: Resistance and Endurance exercise After ChemoTherapy (REACT), Exercise and Nutrition Routine Improving Cancer Health (ENRICH), and Move More for Life (MM4L). Participants were eligible if they were aged $\geq 18$ years and had completed primary cancer treatment. Physical activity was objectively assessed by accelerometers or pedometers. Participants completed self-reported questionnaires on demographic, psychosocial, and environmental factors. Information regarding clinical factors was obtained from
\end{abstract}

medical records or patient self-report. Multivariable linear regression analyses were applied on the pooled dataset to identify factors that were significantly correlated with physical activity. In addition, the explained variance of the model was calculated.

Results The multivariable regression model revealed that older age, $(\beta=-0.01,95 \% \mathrm{CI}=-0.02 ;-0.003)$, higher body mass index $(\beta=-0.05,95 \% \mathrm{CI}=-0.06 ;-0.03)$, lower self-efficacy $(\beta=0.2,95 \% \mathrm{CI}=0.08 ; 0.2)$, and less social support $(\beta=0.1,95 \% \mathrm{CI}=0.05 ; 0.2)$ were significantly correlated with lower physical activity. This model explained $15 \%$ of the variance in physical activity.

Conclusion Age, body mass index, self-efficacy, and social support were significantly correlated with objectively assessed physical activity in breast cancer survivors. It may therefore be recommended that physical activity intervention studies in
Laurien M Buffart

1.buffart@vumc.nl

Caroline S Kampshoff

c.kampshoff@vumc.nl

Fiona Stacey

fiona.stacey@newcastle.edu.au

Camille E Short

camille.short@adelaide.edu.au

Willem van Mechelen

w.vanmechelen@vumc.nl

Mai JM Chinapaw

m.chinapaw@vumc.nl

Johannes Brug

j.brug@vumc.nl

Ronald Plotnikoff

ron.plotnikoff@newcastle.edu.au
Erica L James

erica.james@newcastle.edu.au

1 Department of Public and Occupational Health and the EMGO Institute for Health and Care Research, VU University Medical Center, Amsterdam, the Netherlands

2 School of Medicine and Public Health, Priority Research Centre for Health Behaviour, Priority Research Centre in Physical Activity and Nutrition, and Hunter Medical Research Institute, University of Newcastle, Callaghan, NSW, Australia

School of Medicine, University of Adelaide, Adelaide, Australia

4 Department of Epidemiology and Biostatistics and the EMGO Institute for Health and Care Research, VU University Medical Center, De Boelelaan 1089a, 1081 HV Amsterdam, The Netherlands

School of Education and Arts, Priority Research Centre for Physical Activity and Nutrition, University of Newcastle, Callaghan, NSW, Australia 
these women target those who are older, and have a higher body mass index, and should operationalize behavior change strategies designed to enhance self-efficacy and social support.

Trial registration The REACT study is registered at the Netherlands Trial Register [NTR2153]. The ENRICH study is registered at Australian New Zealand Clinical Trials Register [ANZCTRN12609001086257]. And the $M M 4 L$ study is registered at Australian New Zealand Clinical Trials Register [ACTRN12611001061921]

Keywords Breast cancer · Physical activity · Correlates . Accelerometers $\cdot$ Pedometers

\section{Introduction}

Physical activity (PA), both during and after cancer treatment, has shown to improve cardiorespiratory fitness [1] and enhance quality of life [2] and has been associated with improved survival in breast cancer survivors (BCS) [3]. Therefore, BCS are advised to avoid physical inactivity, encouraged to engage in aerobic exercises for at least $150 \mathrm{~min}$ per week and include resistance exercises twice a week [4]. However, shortly after diagnosis, PA tends to decline in many BCS [5], and consequently, most survivors have insufficient levels of PA [6].

Given the beneficial effects of PA on health outcomes, effective interventions to obtain and maintain sufficient PA among BCSs should be pursued. Identifying modifiable and unmodifiable correlates of PA in BCS may facilitate the development of PA interventions. Modifiable correlates (e.g., psychosocial) provide insights of intervention components (i.e., mediators) via which PA improvement might be achieved. Non-modifiable correlates, such as demographics (e.g., age) or clinical variables (e.g., treatment type) indicate which subgroups of patients are most at risk for physical inactivity and can thus help to identify relevant target populations for intervention. Theoretical behavior change frameworks such as the social cognitive theory [7] and socioecological models [8] strongly recommend taking a broad range of correlates into account while investigating PA behavior, including demographic, clinical, psychosocial, and environmental factors.

Previous studies that have examined correlates of selfreported PA among BCS found a significant association of older age [9, 10], higher body mass index (BMI) [9], lower education level [11], and lower social support [10] with lower PA. Self-reported measures of PA have a predominant position in the existing literature, yet these instruments are prone to overreporting due to social desirability bias and/or misinterpretation of the survey questions [12].
Objective PA monitoring using accelerometers or pedometers overcomes these limitations and provides a more valid estimate of PA as well as more precise associations with potential correlates [13]. Few recent studies have examined correlates of objectively assessed PA in cancer survivors [14-16]. Boyle et al. reported that demographic factors including older age and lower level of education and clinical factors including no family history of breast cancer were associated with lower PA in BCS [14]. Additionally, in colon cancer and non-Hodgkin lymphoma survivors, older age and higher BMI or larger waist circumference were found to be significantly associated with lower PA [15, 16]. So while some correlates identified via self-report and objective assessment correspond, there are too few studies in BCS with objective assessments to draw firm conclusions.

To facilitate the development of effective and targeted interventions aiming to improve PA among BCS, we aimed to identify demographic, clinical, psychosocial, and environmental correlates of objectively assessed PA in a large group of BCS.

\section{Methods}

\section{Study design}

The current study has a cross-sectional design and to ensure a large sample size, we utilized baseline data from BCS who had participated in three different intervention studies, i.e., the Resistance and Endurance exercise After ChemoTherapy (REACT) study [17]; Exercise and Nutrition Routine Improving Cancer Health (ENRICH) study [18], and Move More for Life $(M M 4 L)$ study [19] $\left(\mathrm{n}_{\text {total }}=574\right)$. All three studies were randomized controlled trials that evaluated the effectiveness of a PA or a healthy lifestyle intervention on objectively assessed PA as outcome measure, compared to usual care or a wait list control group. The REACT study was approved by the Medical Ethics Committee of the VU University Medical Centre. The ENRICH and MM4L studies were approved by the Human Research Ethics Committee of the University of Newcastle (ENRICH: H-2009-0347 and MM4L: H-2010-11-3). Full details of the three study designs including the recruitment strategy utilized, inclusion and exclusion criteria, have been described in earlier protocol papers [17-19] and summarized in Fig. 1.

\section{Eligibility criteria}

The current analysis included female BCS aged $\geq 18$ years who had completed primary cancer treatment. Exclusion criteria were (1) being unable to perform basic activities such as self-care and walking, (2) having other serious diseases that 


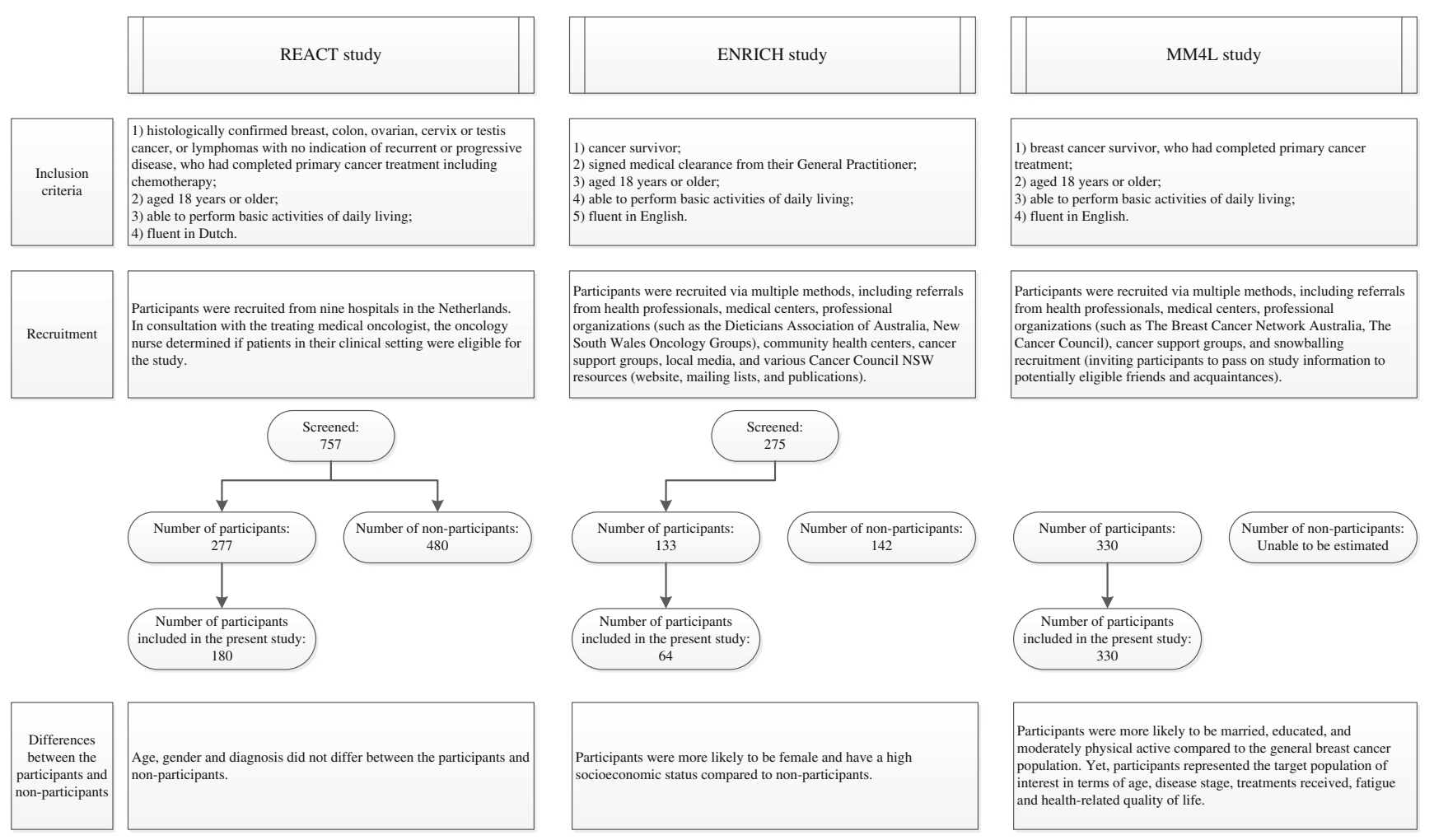

Fig. 1 Inclusion criteria, recruitment strategies, and differences between participants and non-participants of the included studies

hamper PA (e.g., heart failure and cognitive disorders), and (3) being unable to understand and read the first language of the country of recruitment. Participants in the REACT study were recruited from nine hospitals in the Netherlands. In consultation with the treating medical oncologist, the oncology nurse determined if patients in their clinical setting were eligible for the study. Participants in the ENRICH and MM4L studies were recruited via community advertising, health professional, cancer charity, and self-referral (Fig. 1).

\section{Physical activity}

In the REACT study, objective PA was assessed using accelerometers (ActiTrainer; Actigraph, Fort Walton Beach Florida, USA). This is a lightweight PA monitor measuring PA using vertical accelerations that were converted into activity counts per minute (cpm; sum of counts for y-axis, divided by the number of days worn). Raw data was recorded in epochs of $60 \mathrm{~s}$ and non-wearing time was defined as $90 \mathrm{~min}$ of consecutive zero counts. Accelerometer data were processed using ActiLife software version 6.10.2 (ActiGraph, Pensacola, Florida, USA). The ENRICH and MM4L studies used pedometers (Yamax Digi-Walker, SW200) to assess PA. A pedometer is also a lightweight device that assesses daily step counts (sum of steps, divided by the number of days worn). Participants were instructed to wear the accelerometer/pedometer at the hip for seven consecutive days during all waking hours. Both PA monitors are recognized as reliable and valid tools to objectively assess PA in adults [20, 21] and have been used in previous studies among cancer survivors [22]. While accelerometers provide more detailed information on PA than pedometers do, pedometers are much less expensive and therefore more financially feasible for larger studies [23]. Strong convergent validity between accelerometers and pedometers has been demonstrated [13].

Despite the common use of accelerometers and pedometers, a standardized way to process and summarize the collected data is currently lacking. Following the suggestions of Masse et al., we calculated within our own datasets the minimum number of days needed to obtain a reliable measure of objectively measured PA [24]. First, we computed the between-day intraclass correlation coefficient and $95 \%$ confidence interval (CI). Next, we calculated the required days of monitoring needed to achieve reliabilities of $0.70,0.80$, and 0.90 respectively, by using the between-day intraclass correlation coefficient according to the Spearman-Brown prophecy formula [25]. To obtain $75 \%$ reliability, the minimum number of days needed for the accelerometer (REACT data) and pedometer data (MM4L data) was 5 days in both studies, in which a valid day of wearing time was defined as $10 \mathrm{~h}$. We were unable to apply this data reduction rule to the data of the 
ENRICH study; the pedometers in this study were sealed and therefore day-specific step counts were not available.

\section{Demographic factors}

The demographic factors were collected using a self-report questionnaire and included age at baseline (in years), marital status $(0=$ no partner; $1=$ married or de facto), education $(0=$ low/intermediate (i.e., elementary, and lower and secondary vocational education); $1=$ high (i.e., higher vocational and university education)), and employment status $(0=$ no paid (i.e., retired, unemployed, household duties, or student); 1 = paid (i.e., full-time or part-time)).

\section{Clinical factors}

Clinical information was retrieved from medical records (REACT study) or collected using self-report questionnaires (ENRICH study and MM4L study) and included previous treatment with chemotherapy, surgery, radiation therapy, immunotherapy, hormone therapy, history of cancer (i.e., a previous cancer diagnosis prior to most recent diagnosis), and time since breast cancer diagnosis (in months). The number of comorbidities was the sum of each of the following conditions: heart disease, lung disease, diseases of the digestive system, diseases of the nervous system, endocrine disease, mental disorder, rheumatism or arthritis, chronic pain, and other conditions. Furthermore, participants' BMI was calculated from measured body weight and height. In the REACT study, the participants' body weight and height was measured by a health professional. In the ENRICH study and MM4L study, participants were asked to measure and report their own current body weight and height. Clinical measurements and self-report body weight and height have shown strong correlations between each other; however, self-reports may overestimate height and underestimate weight [26].

\section{Psychosocial factors}

Self-efficacy. In all three studies, self-efficacy was assessed with the following question: "How confident are you that you will be physically active in the following situations?" In the REACT study, participants were asked to respond on a 10-point Likert-type scale, and the following five situations were described: feeling tired; bad mood; do not have the time; on vacation; bad weather (Cronbach's $\alpha=0.80$ ). This five-item questionnaire has been reported as a reliable outcome measure [27]. In the ENRICH study, nine situations were rated on a 5-point Likert scale: feeling tired; bad mood or feeling depressed; when you have to do it by yourself; when it becomes boring; there are no noticeable improvements in fitness; having other demands; feeling stiff or sore; bad weather; or having to get up early even on weekends (Cronbach's $\alpha=0.89$ ). This nine-item questionnaire has demonstrated validity and reliability for use in a population of diabetes patients [28]. In the MM4L study, the following 12 situations were rated on a 5-point Likert-type scale: feeling tired; a little ill; little stiff or sore; bad mood or feeling depressed; when you have to do it by yourself; bad weather; lacking discipline; not a priority; lacking time; not enjoying exercise; no encouragements to exercise; there are no noticeable improvements in fitness (Cronbach's $\alpha=0.94$ ). Two previous self-efficacy scales were combined in the MM4L study; and reliability and validity of both scales have been established [28, 29]. For all studies, a higher score indicated higher self-efficacy scores of the individual.

Social support. In the REACT study, social support was assessed using a questionnaire with the statement "The following people are supportive of my regular PA," followed by the following persons: family; friends; coworkers; and other cancer patients (Cronbach's $\alpha=0.92$ ). The participants were asked to score how much they agreed with the statement for each person on a 5-point Likert-scale. In the ENRICH study, social support was assessed using a two-item questionnaire. Participants were asked to rate on a 5-point Likert scale whether people in their social network are likely to help them participate in regular PA, and whether they felt that someone in their social network will provide the support they need in order to be regularly physically active (Cronbach's $\alpha=0.90$ ). In the MM4L study, social support was assessed using a questionnaire starting with the statement "During the past 4 months, my family and friends," followed by the following suggestions: exercised with me; encouraged me to stick with my program; changed their schedule to exercise together; offered to exercise with me; reminded me to exercise; planned exercise on recreational outings; discussed exercise with me; talked about exercise; plan activities around exercise; asked me for ideas on exercise; took over chores; made positive comments about my physical appearance; got angry at me for exercising; criticized me for exercising; gave me rewards for exercising (Cronbach's $\alpha=0.92$ ). Participants were asked to score how often they perceived social support from their friends and family on a 5-point Likert-type scale (1, none to 5, very often). The social support questionnaire included in the MM4L study is a valid and reliable instrument for perceived social support in adults, specific to health-related exercise behaviors [30]. The social support questionnaires included in the REACT and ENRICH studies are frequently used questionnaires among cancer survivors [31]. For all studies, a higher score indicated a higher social support.

Outcome expectations. In the REACT study, outcome expectations was assessed using an eight-item scale starting with the statement: "When I am physically active, then I will," followed by eight suggestions including increase my health; feel better about myself; be more physical fit; improved performance of daily activities; lose weight; meet new people; 
feel better and increase my well-being; cope better with stress (Cronbach's $\alpha=0.88$ ). Participants were asked to score how much they agreed with each statement on a 5-point Likert-type scale. In both ENRICH and MM4L studies, the questionnaire on outcome expectations started with "To what extent do you agree or disagree that participating in regular PA in the next 8 weeks would do for you", followed with five statements which included help reduce tension or manage stress; feel more confident about my health; have better sleep; a more positive outlook; control weight (ENRICH Cronbach's $\alpha=0.92 ; M M 4 L$ Cronbach's $\alpha=0.80)$. Participants were asked to score how much they agreed with each statement on a 5-point Likert scale. For all studies, higher scores on outcome expectations indicated higher expectations of perceived benefits.

\section{Environmental factor-geographic location}

In the current study, geographic location was dichotomized into currently living in an urban area versus living in rural or remote areas. In the REACT study and ENRICH study, classification was based on country-defined zip codes. In the $M M 4 L$ study, participants were asked to report whether they were currently living in a city or in a rural/remote area.

\section{Data treatment and synchronization of variables across studies}

To synchronize variables across studies, the following conversion procedure was applied: (1) the negatively keyed items of the $M M 4 L$ social support questionnaire were reverse-coded; (2) the sum scores of the three psychosocial questionnaires were linearly transformed to a 0-100 scale; (3) the normality assumption was tested, and three continuous variables were found to be highly skewed. Consequently, we dichotomized comorbidity into the presence (1) or absence (0) of two or more comorbidities, and time since diagnosis into longer (1) or shorter (0) than 3 years ago. Outcome expectations was categorized into four groups based on quartiles; (4) PA, self-efficacy, and social support were normally distributed; however, because different outcome measurements were used across studies, we computed standardized or " $z$ "-scores (mean $=0$ and a standard deviation $=1$ ) in each study by subtracting the mean score at baseline from the individual score, divided by the mean standard deviation at baseline; (5) transformation of the variable names of the original studies into current project's coding scheme; and (6) export of all variables of interest into a final data file for the proposed statistical analyses.

\section{Statistical analyses}

Descriptive statistics (mean and standard deviation (SD)) were calculated for all study variables. Univariable and multivariable linear regression analyses were conducted on the pooled data to identify factors significantly correlated with PA. The potential correlates of PA were regressed on PA ("z"-transformed). By default, study as covariate was retained in the univariable and multivariable models to account for varying sample size and clustering of data within the studies. The multivariable regression analyses included a backward selection procedure in which factors with the highest $p$ value were removed from the model one by one. Only variables with a $p$ value of $\leq 0.05$ were retained in the final multivariable model. The unstandardized and standardized regression coefficients $(\beta)$ with $95 \% \mathrm{CI}$ and the explained variance $\left(R^{2}\right)$ of the model were reported accordingly. Due to z-transformations, interpretations of the regression models were based on standardized $\beta$ 's. Prior to the multivariable regression analyses, the possibility of multicollinearity between the potential correlates was checked. Because the variance inflation factors of the included factors were small $(\leq 2)$, we concluded that multicollinearity would not occur in the final model. The statistical analyses were performed using Statistical Package of Social Sciences (SPSS, Inc., Evanston, IL) version 20.0.

\section{Results}

\section{Baseline characteristics}

In total, 484 BCS provided complete baseline data on all variables of interest $(84 \%$ of the total sample $(n=574))$. There were no significant differences $(p>0.05)$ between women with complete data and 90 women with incomplete baseline data on PA or the potential correlates of PA, except for marital status (i.e., participants with a partner were more likely to have complete baseline data). Participants were on average 54.5 years old (SD 9.2); $77 \%$ had a partner, $43 \%$ had completed a bachelor or master degree, and $60 \%$ were employed (Table 1). At least $95 \%$ of the participants underwent surgery to treat breast cancer, revealing very little variability within our population; therefore, we omitted surgery as a potential correlate of PA from the multivariable regression analyses. In the REACT study, an average (SD) of 252.3 (97.8) activity cpm were measured. The ENRICH study and MM4L study reported mean (SD) step counts of 8406.2 (3485.5) and 7694.8 (2831.6), respectively (Table 1).

\section{Correlates of PA}

The results of the univariable and multivariable linear regression analyses are presented in Table 2 . The multivariable regression model showed significant associations for higher age $(\beta=-0.01,95 \% \mathrm{CI}=-0.02 ;-0.003)$ and higher $\mathrm{BMI}$ $(\beta=-0.05,95 \% \mathrm{CI}=-0.06 ;-0.03)$ and lower self-efficacy $(\beta=0.2,95 \% \mathrm{CI}=0.08 ; 0.2)$ and lower social support 
Table 1 Baseline characteristics

\begin{tabular}{|c|c|c|c|c|}
\hline & $\begin{array}{l}\text { REACT } \\
n=180\end{array}$ & $\begin{array}{l}\text { ENRICH } \\
n=64\end{array}$ & $\begin{array}{l}\text { MM4L } \\
n=330\end{array}$ & $\begin{array}{l}\text { Pooled } \\
n=574\end{array}$ \\
\hline \multicolumn{5}{|l|}{ Physical activity } \\
\hline $\begin{array}{l}\text { Mean daily activity counts, mean (SD) } \\
\text { Mean daily steps, mean (SD) }\end{array}$ & $252.3(97.8)$ & $8406.2(3485.5)$ & $7694.8(2831.6)$ & \}z-score \\
\hline \multicolumn{5}{|l|}{ Demographic } \\
\hline Age, mean (SD) (years) & $51.8(9.4)$ & $54.8(11.0)$ & $55.9(8.3)$ & $54.5(9.2)$ \\
\hline Married or de facto $(n(\%))$ & $148(82)$ & $41(64)$ & $250(77)$ & $439(77)$ \\
\hline \multicolumn{5}{|l|}{ Education } \\
\hline Low/intermediate $(n(\%))$ & $106(60)$ & $34(54)$ & $184(56)$ & $324(57)$ \\
\hline $\operatorname{High}(n(\%))$ & $72(40)$ & $29(46)$ & $146(44)$ & $247(43)$ \\
\hline \multicolumn{5}{|l|}{ Being employed, } \\
\hline Employed, $(n(\%))$ & $121(67)$ & $32(51)$ & $189(57)$ & $342(60)$ \\
\hline Not employed, $(n(\%))$ & $59(33)$ & $31(49)$ & $141(43)$ & $231(40)$ \\
\hline \multicolumn{5}{|l|}{ Clinical } \\
\hline \multicolumn{5}{|l|}{ Type of treatment, } \\
\hline Chemotherapy (yes) $(n(\%))$ & $180(100)$ & $52(85)$ & $231(70)$ & $463(81)$ \\
\hline Radiation therapy (yes) $(n(\%))$ & $124(69)$ & $49(85)$ & $225(68)$ & $398(70)$ \\
\hline Surgery (yes) $(n(\%))$ & $177(98)$ & $62(97)$ & $306(93)$ & $545(95)$ \\
\hline Immunotherapy (yes) $(n(\%))$ & $35(19)$ & 0 & $15(5)$ & $50(9)$ \\
\hline Hormone therapy (yes) $(n(\%))$ & $125(69)$ & $45(82)$ & $201(61)$ & $371(66)$ \\
\hline $\begin{array}{l}\text { Time between date of diagnosis and } \\
\text { baseline, mean (SD) (months) }\end{array}$ & $7.9(1.5)$ & $44.2(83.5)$ & $69.0(50.9)$ & $47.6(55.1)$ \\
\hline History of cancer (yes) $(n(\%))$ & $15(8)$ & $4(7)$ & $19(6)$ & $38(7)$ \\
\hline Sum of comorbidities, mean (SD) & $0.5(0.7)$ & $2.3(1.7)$ & $1.7(1.5)$ & $1.4(1.5)$ \\
\hline BMI $\left(\mathrm{kg} / \mathrm{m}^{2}\right)$, mean $(\mathrm{SD})$ & $27.2(4.8)$ & $27.0(6.1)$ & $26.9(5.2)$ & $27.0(5.2)$ \\
\hline \multicolumn{5}{|l|}{ Psychosocial (Range 0-100) } \\
\hline Self-efficacy, mean (SD) & $60.0(16.7)$ & $52.5(17.8)$ & $50.7(22.3)$ & z-score \\
\hline Social support, mean (SD) & $80.3(20.4)$ & $48.8(33.2)$ & $30.1(15.6)$ & z-score \\
\hline Outcome expectations, mean (SD) & $76.4(14.7)$ & $73.4(16.6)$ & $81.8(13.9)$ & $79.2(14.8)$ \\
\hline \multicolumn{5}{|l|}{ Environmental } \\
\hline Urban area, (yes) $(n(\%))$ & $45(25)$ & $64(100)$ & $155(47)$ & $264(46)$ \\
\hline
\end{tabular}

$S D$ standard deviation, $n$ number

$(\beta=0.1,95 \% \mathrm{CI}=0.05 ; 0.2)$ with lower PA. Based on the standardized $\beta$ 's, BMI had the strongest association with PA. The final model explained $15 \%$ of the variance in PA. No significant correlations were found between treatment-related characteristics or geographic location and PA.

\section{Discussion}

The current study examined possible demographic, clinical, psychosocial, and environmental correlates of objectively assessed PA in a large group of BCS. We found that age, BMI, self-efficacy and social support were significantly correlated with $\mathrm{PA}$.

Our finding that older age was significantly associated with lower PA is in line with previous research in BCS using self-reported PA questionnaires [9, 10]. Demographic factors, such as age, are non-modifiable factors and highlight those subpopulations that are more likely to be physically inactive and may thus have a greater need for intervention. To date, previous studies predominately reached younger BCS, and consequently, PA interventions in older female BCS remain understudied [32]. Future studies should develop PA interventions appealing to older BCS and evaluate the effectiveness of such interventions in this subpopulation.

Higher BMI was also found to be significantly associated with lower objectively assessed PA. This finding supports previous studies using subjective and objective PA assessments [33]. The current cross-sectional study design does not allow us to detangle the causal direction of the association between BMI and PA (i.e., whether BCS with higher BMI have lower PA or vice versa, BCS with lower PA have higher 
Table 2 Unstandardized and standardized regression coefficients and their $95 \%$ confidence intervals as results from univariable and multivariable regression analyses with PA z-scores as dependent variable and demographic, clinical, psychosocial, and environmental variables as independent variables

\begin{tabular}{|c|c|c|c|}
\hline & $\begin{array}{l}\text { Univariable } \\
\text { Unstandardized } \\
\beta(95 \% \mathrm{CI})\end{array}$ & $\begin{array}{l}\text { Multivariable } \\
\text { Unstandardized } \\
\beta(95 \% \mathrm{CI})\end{array}$ & $\begin{array}{l}\text { Multivariable } \\
\text { Standardized } \\
\beta\end{array}$ \\
\hline \multicolumn{4}{|l|}{ Demographic } \\
\hline Age (years) & $-0.01(-0.02 ;-0.01)^{*}$ & $-0.01(-0.02 ;-0.003)^{*}$ & -0.11 \\
\hline Married or de facto & $0.1(-0.1 ; 0.3)$ & & \\
\hline Education & $0.1(-0.1 ; 0.3)$ & & \\
\hline Being employed & $0.1(-0.1 ; 0.3)$ & & \\
\hline \multicolumn{4}{|l|}{ Clinical } \\
\hline \multicolumn{4}{|l|}{ Type of treatment } \\
\hline Chemotherapy & $-0.1(-0.3 ; 0.1)$ & & \\
\hline Radiation therapy & $-0.2(-0.4 ; 0.01)$ & & \\
\hline Immunotherapy & $0.1(-0.3 ; 0.4)$ & & \\
\hline Hormone therapy & $0.1(-0.1 ; 0.3)$ & & \\
\hline Time between diagnosis and baseline & $0.1(-0.02 ; 0.3)$ & & \\
\hline History of cancer & $-0.1(-0.5 ; 0.2)$ & & \\
\hline Presence of two or more comorbidities & $-0.3(-0.4 ;-0.1)^{*}$ & & \\
\hline BMI $\left(\mathrm{kg} / \mathrm{m}^{2}\right)$ & $-0.1(-0.1 ;-0.04)^{*}$ & $-0.05(-0.06 ;-0.03)^{*}$ & -0.25 \\
\hline \multicolumn{4}{|l|}{ Psychosocial } \\
\hline Self-efficacy & $0.2(0.1 ; 0.3)^{*}$ & $0.2(0.08 ; 0.2)^{*}$ & 0.17 \\
\hline Social support & $0.2(0.1 ; 0.3)^{*}$ & $0.1(0.05 ; 0.2)^{*}$ & 0.13 \\
\hline \multicolumn{4}{|l|}{ Outcome expectations } \\
\hline quartile 1 vs. 2 & $0.1(-0.1 ; 0.3)$ & & \\
\hline quartile 1 vs. 3 & $-0.1(-0.3 ; 0.2)$ & & \\
\hline quartile 1 vs. 4 & $0.02(-0.2 ; 0.3)$ & & \\
\hline \multicolumn{4}{|l|}{ Environmental } \\
\hline Urban area & $0.01(-0.2 ; 0.2)$ & & \\
\hline
\end{tabular}

$\beta$ regression coefficients, $C I$ confidence interval;

$* p \leq 0.05$
BMI). Nevertheless, the current study underlines the importance of targeting overweight and obese BCS. Such PA interventions for overweight and obese BCS may also contribute to achieving and maintaining weight loss in cancer survivors, especially when combined with a dietary intervention [34]. This may particularly be important given the independent relationships between obesity and breast cancer recurrence [35] as well as inactivity and breast cancer recurrence [36] reported in observational studies.

We found that lower self-efficacy to overcome PA-related barriers was significantly associated with lower PA. This finding is consistent with previous studies using self-reported PA questionnaires in a mixed group of cancer survivors [37, 38]. Hence, interventions aiming at increased PA levels may be more effective by including strategies to improve the individuals' self-efficacy, especially for BCS who do not feel confident about their abilities to undertake new activities and stay engaged. Motivational interviewing has been identified as a promising strategy to improve a person's self-efficacy [39] and consequently improve PA. Bennett et al. [40] conducted a randomized controlled trial and reported a significant increase in PA after a motivational interviewing intervention among cancer survivors [40]. They also reported that cancer survivors with lower self-efficacy scores at baseline reported less PA improvement compared to those with higher self-efficacy scores, further indicating that boosting self-efficacy is of great importance. Additional components such as goal setting, skill development, or self-monitoring [41] may also be effective strategies to improve self-efficacy.

Finally, less social support from family and friends was significantly associated with lower PA among our population of BCS. This is in line with a previous review among healthy individuals reporting a strong correlation between less social support from family and friends and lower self-reported PA [42]. Likewise, Pinto et al. reported a significant correlation of social support with self-reported PA among BCS [10]. Yet, studies in other cancer diagnosis such as colon [43] and head and neck [44] and lung cancer [45] reported no significant association of social support with PA. A possible explanation for the differences among the cancer survivor groups may be 
related to gender [46]. On the other hand, despite the use of valid questions [30], the phrasing of the items in the current study might have caused a bias toward a positive association. Future studies should further examine the role of social support in BCS and in which way social support could be successfully promoted in PA interventions.

In contrast with our findings, two previous studies have reported conflicting findings on associations between geographical location (i.e., living in urban versus rural or remote areas) and PA [47, 48]. Weaver et al. showed that rural cancer survivors from the USA were less likely to be physically active compared to urban survivors [47]. Whereas, Lynch et al. found that urban colorectal cancer survivors from Australia were less likely to achieve or maintain a physical healthy lifestyle postdiagnosis compared to rural survivors [48]. Conflicting results may be related to the urban and rural/ remote classification or differences in context or regional and related community factors. Given higher mortality [49] and additional barriers to supportive care $[50,51]$ faced by rural cancer survivors, further exploration of PA correlates for this target group is warranted.

The remaining factors that were considered in the current study showed no significant association with PA, including marital and employment status, level of education, all treatment-related characteristics, and comorbidity. The fact that these non-modifiable factors were not associated with PA suggests that no additional subgroups that are at risk for physical inactivity can be distinguished in BCS, at least not based on these variables included in the present study. Our findings on those remaining variables are in line with previous research; however, the current literature on correlates of PA among BCS is limited.

Our final multivariable model explained $15 \%$ of the variance in PA, indicating that there may be other variables explaining PA behavior that were not included in the current study are important to explain differences in PA among BCS. The current findings on self-efficacy and social support may suggest a role for behavioral theory such as social cognitive theory in developing interventions for BCS. Theory-based interventions have shown to be more effective in changing behavior than non-theory based interventions. However, current theories do not incorporate potential cancer- or treatment-related factors (e.g., chemotherapy dose and neurotoxic side effects) and there is a need for refining current theories to BCS. Forthcoming studies will benefit from examination of in-depth information on clinical factors and a broader set of contextual or environmental factors (such as the availability of and accessibility to PA opportunities including sidewalks, bicycle lanes, and sports facilities).

The following limitations are worth noting. First, the cross-sectional design of the study does not allow drawing conclusions on causal relationships. Hence, in a previous review among healthy individuals, self-efficacy was found to be the most important mediator [52] of the exercise intervention effect on PA, supporting findings of the current study. Yet, future experimental studies are warranted to investigate the mediating role of psychosocial variables of the intervention effect on PA in cancer survivors. Second, although objective assessments of PA with accelerometers and pedometers are the preferred method to assess PA, they may have underestimated PA because the monitors were worn around the hip and can therefore not accurately assess upper body activities, nor can they account for swimming activities or activities that require extra effort (e.g., walking uphill). However, accelerometers and pedometers are able to adequately distinguish between individuals with higher or lower PA levels [13]. Furthermore, in order to pool data from both PA outcome measures, we computed standardized or Z-scores which hamper the interpretation of the reported effect sizes. Nevertheless, the level of significance and the directions of the reported associations are informative. Third, despite the strong correlations between clinical measurements and self-reported body weight and height [26], self-reports may underestimate weight and overestimate height. Aiming to avoid additional bias, BMI was included as a continuous variable in the current study [53]. Fourth, the definition and impact of living in a rural area compared to a metropolitan area varies enormously between Australia and the Netherlands. Since no current gold standard is available for pooling data from different countries, the use of the urban versus pooled rural/remote classification in the current study was a first attempt to acknowledge the international differences. Last, the participants who provided data for this analysis have been recruited to take part in behavior change trials rather than being drawn from a population sample. Therefore, eligible patients who declined participation may impact the generalizability of the results. Yet, each study checked whether the participants and non-participants differed on demographic and clinical factors [54-56], which revealed that patients who decided to participate were more likely to be higher educated, have a higher socioeconomic status, and be more physically active compared to those who declined participation (Fig. 1) $[55,56]$. Worth noting, the three studies were conducted predominantly among Caucasian women, and therefore, ethnicity was unable to be explored as a possible correlate. As a consequence, the findings of the current study may not be generalizable to other ethnic groups, including ethnic minorities (e.g., indigenous Australians).

In conclusion, we found that female BCS who are older, reported higher BMI scores, lower self-efficacy scores, or less social support from family and friends may be at higher risk of being physically inactive. This information should facilitate the development of targeted interventions aiming to improve PA among BCS. Future studies should gain a better understanding of the specific determinants that are unique to older or heavier BCS, which provides important information for further intervention design. In addition, forthcoming studies 
in BCS should further investigate the effectiveness of behavior change strategies on PA.

Acknowledgments This work represents a collaborative research project which was partly funded by the EMGO+ Institute for Health and Care Research Travel Grant 2013 awarded to CK. We thank Dr. Christopher Oldmeadow from the Hunter Medical Research Institute, NSW, Australia for his statistical support during this project. Furthermore, a special thank you to the survivors who participated in our trials. The authors acknowledge the project advisory groups and all healthcare professionals involved in each individual study.

The REACT study is part of the A-CaRe Program, http://www.a-care. org, supported by Alpe d'HuZes/ Dutch Cancer Society [Grant number ALPE 2009-4619]. The ENRICH study was supported by funding from the Australian Better Health Initiative: A joint Australian, State and Territory government initiative with additional funding and infrastructure support provided by the Cancer Council NSW and Hunter Medical Research Institute. In addition, the authors acknowledge the ENRICH co-investigators: Ms. Kathy Chapman, Professor David Lubans, Dr. Allison Boyes, Ms. Gabrielle Asprey, and Professor Afaf Girgis. The MM4L study was supported by a New South Wales Cancer Institute Research Scholar Award [10/RSA/1-27] awarded to CS and acknowledge co-investigator Professor Afaf Girgis. The authors thank the Cancer Network Australia, Dragon's Abreast, YWCA Encore and Kimberly Parsons for their assistance with recruiting participants for MM4L study.

Author contributions CK, FS, CS, EJ, and LB designed the study. CK, FS and CS drafted the manuscript. CK, FS, EJ, and LB performed statistical analyses. WM, MC, JB, and RP provided critical revision of the manuscript and important intellectual content. All authors read and approved the final manuscript and take responsibility for the integrity of the data.

\section{Compliance with ethical standards}

Conflict of interest The authors declare that they have no competing interests.

Open Access This article is distributed under the terms of the Creative Commons Attribution-NonCommercial 4.0 International License (http://creativecommons.org/licenses/by-nc/4.0/), which permits any noncommercial use, distribution, and reproduction in any medium, provided you give appropriate credit to the original author(s) and the source, provide a link to the Creative Commons license, and indicate if changes were made.

\section{References}

1. Jones LW, Liang Y, Pituskin EN, Battaglini CL, Scott JM, Hornsby WE, et al. (2011) Effect of exercise training on peak oxygen consumption in patients with cancer: a meta-analysis. Oncologist 16: $112-120$

2. Mishra SI, Schrerer RW, Geigle PM, Berlanstein DR, Topaloglu O, Gotay CC, et al. (2012) Exercise interventions on health-related quality of life for cancer survivors. Cochrane Database Syst Rev: CD007566

3. Schmid D, Leitzmann MF (2015) Cardiorespiratory fitness as predictor of cancer mortality: a systematic review and meta-analysis. Ann Oncol 26:272-278
4. Schmitz KH, Courneya KS, Matthews C, Demark-Wahnefried W, Galvao DA, Pinto BM, et al. (2010) American College Of Sports Medicine roundtable on exercise guidelines for cancer survivors. Med Sci Sports Exerc 42:1409-1426

5. Kwan ML, Sternfeld B, Ergas IJ, Timperi AW, Roh JM, Hong CC, et al. (2012) Change in physical activity during active treatment in a prospective study of breast cancer survivors. Breast Cancer Res Treat 131:679-690

6. Littman AJ, Tang MT, Rossing MA (2010) Longitudinal study of recreational physical activity in breast cancer survivors. J Cancer Surviv 4:119-127

7. Bandura A (2004) Health promotion by social cognitive means. Health Educ Behav 31:143-164

8. McLaren L, Hawe P (2005) Ecological perspectives in health research. J Epidemiol Community Health 59:6-14

9. Brunet J, Sabiston CM (2011) Self-presentation and physical activity in breast cancer survivors: the moderating effect of social cognitive constructs. J Sport Exerc Psychol 33:759-778

10. Pinto BM, Trunzo JJ, Reiss P, Shiu SY (2002) Exercise participation after diagnosis of breast cancer: trends and effects on mood and quality of life. Psychooncology 11:389-400

11. Hong S, Bardwell WA, Natarajan L, Flatt SW, Rock CL, Newman VA, et al. (2007) Correlates of physical activity level in breast cancer survivors participating in the Women's healthy eating and living (WHEL) study. Breast Cancer Res Treat 101:225-232

12. Tucker JM, Welk GJ, Beyler NK (2011) Physical activity in U.S.: adults compliance with the physical activity guidelines for Americans. Am J Prev Med 40:454-461

13. Harris TJ, Owen CG, Victor CR, Adams R, Ekelund U, Cook DG (2009) A comparison of questionnaire, accelerometer, and pedometer: measures in older people. Med Sci Sports Exerc 41:13921402

14. Boyle T, Vallance JK, Ransom EK, Lynch BM 2015 How sedentary and physically active are breast cancer survivors, and which population subgroups have higher or lower levels of these behaviors? Support Care cancer

15. Lynch BM, Boyle T, Winkler E, Occleston J, Courneya KS, Vallance JK (2016) Patterns and correlates of accelerometerassessed physical activity and sedentary time among colon cancer survivors. Cancer Causes Control 27:59-68

16. Boyle T, Lynch BM, Ransom EK, Vallance JK (2015) Volume and correlates of objectively measured physical activity and sedentary time in non-Hodgkin lymphoma survivors. Psychooncology

17. Kampshoff CS, Buffart LM, Schep G, van Mechelen W, Brug J, Chinapaw MJ (2010) Design of the Resistance and Endurance exercise After ChemoTherapy (REACT) study: a randomized controlled trial to evaluate the effectiveness and cost-effectiveness of exercise interventions after chemotherapy on physical fitness and fatigue. BMC Cancer 10:658

18. James EL, Stacey F, Chapman K, Lubans DR, Asprey G, Sundquist $\mathrm{K}$, et al. (2011) Exercise and nutrition routine improving cancer health (ENRICH): the protocol for a randomized efficacy trial of a nutrition and physical activity program for adult cancer survivors and carers. BMC Public Health 11:236

19. Short CE, James EL, Girgis A, McElduff P, Plotnikoff RC (2012) Move more for life: the protocol for a randomised efficacy trial of a tailored-print physical activity intervention for post-treatment breast cancer survivors. BMC Cancer 12:172

20. Bassett DR Jr, Ainsworth BE, Leggett SR, Mathien CA, Main JA, Hunter DC, et al. (1996) Accuracy of five electronic pedometers for measuring distance walked. Med Sci Sports Exerc 28:1071-1077

21. Plasqui G, Westerterp KR (2007) Physical activity assessment with accelerometers: an evaluation against doubly labeled water. Obesity (Silver Spring) 15:2371-2379

22. Liu RD, Buffart LM, Kersten MJ, Spiering M, Brug J, van Mechelen W, et al. (2011) Psychometric properties of two physical 
activity questionnaires, the AQuAA and the PASE, in cancer patients. BMC Med Res Methodol 11:30

23. Corder K, Brage S, Ekelund U (2007) Accelerometers and pedometers: methodology and clinical application. Curr Opin Clin Nutr Metab Care 10:597-603

24. Masse LC, Fuemmeler BF, Anderson CB, Matthews CE, Trost SG, Catellier DJ, et al. (2005) Accelerometer data reduction: a comparison of four reduction algorithms on select outcome variables. Med Sci Sports Exerc 37:S544-S554

25. Trost SG, Pate RR, Freedson PS, Sallis JF, Taylor WC (2000) Using objective physical activity measures with youth: how many days of monitoring are needed? Med Sci Sports Exerc 32:426-431

26. Dekkers JC, van Wier MF, Hendriksen IJ, Twisk JW, van Mechelen W (2008) Accuracy of self-reported body weight, height and waist circumference in a Dutch overweight working population. BMC Med Res Methodol 8:69

27. Marcus BH, Selby VC, Niaura RS, Rossi JS (1992) Self-efficacy and the stages of exercise behavior change. Res Q Exerc Sport 63: 60-66

28. Plotnikoff RC, Blanchard CM, Hotz SB, Rhodes R (2001) Validation of the decisional balance scales in the exercise domain from the transtheoretical model: a longitudinal test. Meas Phys Educ Exerc Sci 5:191-206

29. Rogers LQ, Courneya KS, Verhulst S, Markwell S, Lanzotti V, Shah P (2006) Exercise barrier and task self-efficacy in breast cancer patients during treatment. Support Care Cancer 14:84-90

30. Sallis JF, Grossman RM, Pinski RB, Patterson TL, Nader PR (1987) The development of scales to measure social support for diet and exercise behaviors. Prev Med 16:825-836

31. Sherbourne CD, Stewart AL (1991) The MOS social support survey. Soc Sci Med 32:705-714

32. Fong DY, Ho JW, Hui BP, Lee AM, Macfarlane DJ, Leung SS, et al. (2012) Physical activity for cancer survivors: meta-analysis of randomised controlled trials. BMJ 344:e70

33. Lynch BM, Dunstan DW, Healy GN, Winkler E, Eakin E, Owen N (2010) Objectively measured physical activity and sedentary time of breast cancer survivors, and associations with adiposity: findings from NHANES (2003-2006). Cancer Causes Control 21:283-288

34. Travier N, Fonseca-Nunes A, Javierre C, Guillamo E, Arribas L, Peiro I, et al. (2014) Effect of a diet and physical activity intervention on body weight and nutritional patterns in overweight and obese breast cancer survivors. Med Oncol 31:783

35. Chan DS, Vieira AR, Aune D, Bandera EV, Greenwood DC, McTiernan A, et al. (2014) Body mass index and survival in women with breast cancer-systematic literature review and meta-analysis of 82 follow-up studies. Ann Oncol 25:1901-1914

36. Ballard-Barbash R, Friedenreich C, Courneya KS, Siddiqi SM, McTiernan A, Alfano CM (2012) Physical activity, biomarkers, and disease outcomes in cancer survivors: a systematic review. $\mathrm{J}$ Natl Cancer Inst 104:815-840

37. Clark MM, Vickers KS, Hathaway JC, Smith M, Looker SA, Petersen LR, et al. (2007) Physical activity in patients with advanced-stage cancer actively receiving chemotherapy. J Support Oncol 5:487-493

38. Karvinen KH, Courneya KS, Campbell KL, Pearcey RG, Dundas G, Capstick V, et al. (2007) Correlates of exercise motivation and behavior in a population-based sample of endometrial cancer survivors: an application of the theory of planned behavior. Int J Behav Nutr Phys Act 4:21

39. Miller WR, Rollnick S (1991) Motivational interviewing, preparing people to change addictive behavior. The Guildford Press, New York
40. Bennett JA, Lyons KS, Winters-Stone K, Nail LM, Scherer J (2007) Motivational interviewing to increase physical activity in long-term cancer survivors: a randomized controlled trial. Nurs Res 56:18-27

41. Michie S, Abraham C, Whittington C, McAteer J, Gupta S (2009) Effective techniques in healthy eating and physical activity interventions: a meta-regression. Health Psychol 28:690-701

42. Trost SG, Owen N, Bauman AE, Sallis JF, Brown W (2002) Correlates of adults' participation in physical activity: review and update. Med Sci Sports Exerc 34:1996-2001

43. James AS, Campbell MK, DeVellis B, Reedy J, Carr C, Sandler RS (2006) Health behavior correlates among colon cancer survivors: NC STRIDES baseline results. Am J Health Behav 30:720-730

44. Rogers LQ, Courneya KS, Robbins KT, Malone J, Seiz A, Koch L, et al. (2008) Physical activity correlates and barriers in head and neck cancer patients. Support Care Cancer 16:19-27

45. Coups EJ, Park BJ, Feinstein MB, Steingart RM, Egleston BL, Wilson DJ, et al. (2009) Correlates of physical activity among lung cancer survivors. Psychooncology 18:395-404

46. Oliveira AJ, Lopes CS, Rostila M, Werneck GL, Griep RH, Leon AC, et al. (2014) Gender differences in social support and leisuretime physical activity. Rev Saude Publica 48:602-612

47. Weaver KE, Palmer N, Lu L, Case LD, Geiger AM (2013) Ruralurban differences in health behaviors and implications for health status among US cancer survivors. Cancer Causes Control 24: $1481-1490$

48. Lynch BM, Cerin E, Newman B, Owen N (2007) Physical activity, activity change, and their correlates in a population-based sample of colorectal cancer survivors. Ann Behav Med 34:135-143

49. Jong KE, Smith DP, Yu XQ, O'Connell DL, Goldstein D, Armstrong BK (2004) Remoteness of residence and survival from cancer in new South Wales. Med J Aust 180:618-622

50. Williams C, Thorpe R, Harris N, Dickinson H, Barrett C, Rorison F (2006) Going home from hospital: the postdischarge experience of patients and carers in rural and remote Queensland. Aust J Rural Health 14:9-13

51. Paul CL, Hall AE, Carey ML, Cameron EC, Clinton-McHarg T (2013) Access to care and impacts of cancer on daily life: do they differ for metropolitan versus regional hematological cancer survivors? J Rural Health 29(Suppl 1):s43-s50

52. Lewis BA, Marcus BH, Pate RR, Dunn AL (2002) Psychosocial mediators of physical activity behavior among adults and children. Am J Prev Med 23:26-35

53. Preston SH, Fishman E, Stokes A (2015) Effects of categorization and self-report bias on estimates of the association between obesity and mortality. Ann Epidemiol 25:907-911

54. Kampshoff CS, Chinapaw MJ, Brug J, Twisk JW, Schep G, Nijziel MR, et al. (2015) Randomized controlled trial of the effects of high intensity and low-to-moderate intensity exercise on physical fitness and fatigue in cancer survivors: results of the resistance and Endurance exercise after ChemoTherapy (REACT) study. BMC Med 13:275

55. Short CE, James EL, Girgis A, D’Souza MI, Plotnikoff RC (2015) Main outcomes of the move more for life trial: a randomised controlled trial examining the effects of tailored-print and targeted-print materials for promoting physical activity among post-treatment breast cancer survivors. Psychooncology 24:771-778

56. James EL, Stacey FG, Chapman K, Boyes AW, Burrows T, Girgis A, et al. (2015) Impact of a nutrition and physical activity intervention (ENRICH: exercise and nutrition routine improving cancer health) on health behaviors of cancer survivors and carers: a pragmatic randomized controlled trial. BMC Cancer 15:710 